\title{
Arrhythmias and Cardiogenic Shock: A Rare Disease Presentation of Diffuse Large B-Cell Lymphoma with Cardiac Involvement
}

\author{
Sabrina Mariani ${ }^{1}$, Sabrina Pelliccia ${ }^{1,2}$, Maria Paola Bianchi ${ }^{1}$, Monica Piedimonte ${ }^{1,2}$, Martina Bongiovanni ${ }^{3}$, \\ Marco Testa ${ }^{3}$, Arianna Di Napoli ${ }^{4}$ (D) and Agostino Tafuri ${ }^{1,2, *}$
}

1 Hematology, "Sant' Andrea" University Hospital, Sapienza University of Rome, 00189 Rome, Italy; sa.mariani@uniroma1.it (S.M.); sabrina.pelliccia@virgilio.it (S.P.); mariapaolabianchi82@gmail.com (M.P.B.); monica.piedimonte@uniroma1.it (M.P.)

2 Department of Clinical and Molecular Medicine, Sapienza University of Rome, 00189 Rome, Italy

3 Cardiology Unit, Sant' Andrea University Hospital, 00189 Rome, Italy; martina.bongiovanni@gmail.com (M.B.); marcotestaroma@gmail.com (M.T.)

4 Pathology Unit, Department of Clinical and Molecular Medicine, Sant'Andrea Hospital, Sapienza University, 00189 Rome, Italy; arianna.dinapoli@uniroma1.it

* Correspondence: agostino.tafuri@uniroma1.it

Citation: Mariani, S.; Pelliccia, S.; Bianchi, M.P.; Piedimonte, M.; Bongiovanni, M.; Testa, M.; Di Napoli, A.; Tafuri, A. Arrhythmias and Cardiogenic Shock: A Rare Disease Presentation of Diffuse Large B-Cell Lymphoma with Cardiac Involvement. Hemato 2021, 2, 353-357. https://doi.org/10.3390/ hemato2020021

Academic Editor: Carmelo

Carlo-Stella

Received: 10 May 2021

Accepted: 31 May 2021

Published: 1 June 2021

Publisher's Note: MDPI stays neutral with regard to jurisdictional claims in published maps and institutional affiliations.

Copyright: (c) 2021 by the authors. Licensee MDPI, Basel, Switzerland. This article is an open access article distributed under the terms and conditions of the Creative Commons Attribution (CC BY) license (https:// creativecommons.org/licenses/by/ $4.0 /)$.

\begin{abstract}
Extranodal involvement of non-Hodgkin lymphoma (NHL) has been reported in 20-40\% of patients and has been typically observed in the skin, bones, gastrointestinal tract, liver and brain. Cardiac involvement has been reported in up to $20 \%$ of autopsy cases of patients with NHL and accounts for about $2 \%$ of all cardiac malignancies. Here, we report a peculiar case of a secondary cardiac diffuse large B-cell lymphoma (DLBCL), occurring with an abrupt hemodynamic instability, characterized by a sudden ventricular tachycardia and cardiogenic shock. The patient promptly started the first cycle of chemotherapy and was admitted to the cardiac intensive care unit (CICU) of our institution to prevent potential cardiovascular complications during treatment. We applied a fractionated treatment approach, progressively reaching standard doses, to decrease the risk of early death and ensure a successful management.
\end{abstract}

Keywords: diffuse large B-cell lymphoma; cardiac involvement; fractionated chemotherapy

\section{Introduction}

DLBCL is the most frequent lymphoma associated with extranodal involvement with an incidence of 1.7 cases per 100,000 person-years [1]. A reduced 5-year survival rate in patients with DLBCL and extranodal site involvement has been reported in comparison with those who do not present extranodal involvement, at 56 and 61\%, respectively. Moreover, cardiac NHL involvement is often misdiagnosed, and, therefore, it is associated with a poor outcome because of delayed treatment. Reports on these patients are limited due to the lack of evidence-based data and controlled studies in which the diagnostic work-up and therapeutic management are well established. Here, we report a case of secondary cardiac DLBCL promptly diagnosed by multidisciplinary assessment, receiving a fractionated and tailored chemotherapy approach and achieving disease remission without complications.

\section{Case Report}

A 72-year-old man, with a medical history of tachyarrhythmia not further evaluated and diagnosed as atrial fibrillation, not in anticoagulant therapy that occurred in the last two months and in the absence of other cardiovascular comorbidities, presented to ER due to severe dyspnoea without chest pain, agitation and sweating; the patient's blood pressure was not detectable, and he abruptly developed a cardiogenic shock. An electrical 
cardioversion was successfully performed in an emergency because of ventricular tachycardia. The blood count was normal (hemoglobin $11.7 \mathrm{~g} / \mathrm{dL}, \mathrm{MCV} 90 \mathrm{fL}$, white blood cells $7270 / \mathrm{mmc}$, neutrophils $4210 / \mathrm{mmc}$, lymphocytes $1670 / \mathrm{mmc}$, platelets $260.000 / \mathrm{mmc}$ ), while serum tests showed a higher level of lactate dehydrogenase (520 U/L, range 125-225) and brain natriuretic peptide (BNP) $(135.4 \mathrm{pg} / \mathrm{mL}$, range 0-56.00). The troponin I level was normal $(6 \mathrm{pg} / \mathrm{mL})$. The chest X-ray showed a mediastinal enlargement and bilateral pleural effusion. The transthoracic echocardiogram (TTE) highlighted a large mass involving the right atrium and a diffuse thickening of both the right atrium and ventricle; the left ventricle size was normal and the ejection fraction was $56 \%$, with minimal pericardial detachment (Figure 1). However, the day after, while still in the ER, the patient experienced a second episode of ventricular tachycardia, which was treated, this time, with medical therapy. Two days after, he was admitted to the CICU. The total body computed tomography (CT) scan showed a bulky mediastinal mass (transversal diameter $12 \mathrm{~cm}$ ), which induced an encasement of the aortic arch and epiaortic vessels, causing a loss of the anatomical level of cleavage with the pericardium and the right sections of the heart, as well as the right coronary artery. No further lymphadenopathies were documented. As a result of these tests, a CT-guided mediastinal biopsy was performed after obtaining patient stabilization with intravenous antiarrhythmic therapy, under continuous cardiac rhythm monitoring. Histological examination showed infiltration by a non-germinal center B-cell (non-GCB) type DLBCL CD20+, CD79a+, CD30-, CD23-, CD10-, BCL6+, MUM1+, with double expression of BCL2 $(>50 \%)$ and MYC $(>40 \%)$ and a high proliferation index $(\mathrm{Ki}-67=85 \%)$. Break-apart fluorescence in situ hybridization (FISH) assays for MYC, BCL2 and BCL6 genes revealed BCL6 rearrangement. According to the extensive cardiac involvement, careful management of the patient was ensured during all cycles of treatment by a fractionated chemotherapy approach. In fact, the first cycle of the R-COMP (rituximab, vincristine, cyclophosphamide, liposomal doxorubicin and prednisone) chemotherapy regimen was fractionated as described below. The standard vincristine (VCR) dose was reduced to $50 \%$ of the standard dosage $(1.4 \mathrm{mg} / \mathrm{mq})$ on day one. The rituximab dosage $(375 \mathrm{mg} / \mathrm{mq})$ was fractionated in three consecutive days. On day eight, the remaining VCR dose was administered. Finally, on day nine, the full dose of cyclophosphamide $(750 \mathrm{mg} / \mathrm{mq})$ and $50 \%$ of the liposomal doxorubicin $(50 \mathrm{mg} / \mathrm{mq}$ ) were administered. Prednisolone was administered unchanged at $60 \mathrm{mg} / \mathrm{mq}$ on days $1-5$. The risk of full-dose chemotherapy was essentially related to myocardial rupture or life-threatening arrhythmias secondary to rapid tumor lysis. Prophylactic measures for tumor lysis syndrome with fluid management, diuretics and hypouricemic agents were administered. During the entire treatment, the patient remained in CICU with continuous cardiac monitoring, considering the risk of a myocardial perforation. During chemotherapy, no significant arrhythmias occurred. After the first cycle, a TTE performed on the patient showed a mild dimensional reduction of the mass infiltrating the heart, with a left ventricular ejection fraction of $65 \%$. A week later, the patient was discharged. The following cycles of chemotherapy were administered as outpatient treatment every 21 days as scheduled. After the third cycle, an interim whole CT scan performed on the patient showed a reduction of $>50 \%$ of the disease; a TTE performed at the same time documented a complete disappearance of the lymphoma tissue previously detected, with stable left ventricular systolic function. The patient was treated with six R-COMP-21 cycles followed by two further administrations of rituximab. During all chemotherapy sessions, no other arrythmias occurred. ECG was performed after the third cycle contextually at the TTE. A final assessment of the disease included an 18F-fluorodeoxyglucose positron emission tomography and a CT scan (FDG-PET/TC), which showed a complete remission (CR). Furthermore, a cardio CT, performed only at the end of treatment, confirmed a complete remission of cardiac infiltration, and the coronary arteries were undamaged. Echocardiographic follow-up was performed during and after treatment, showing progressive reduction of the mass. The left ventricular ejection fraction remained normal. 


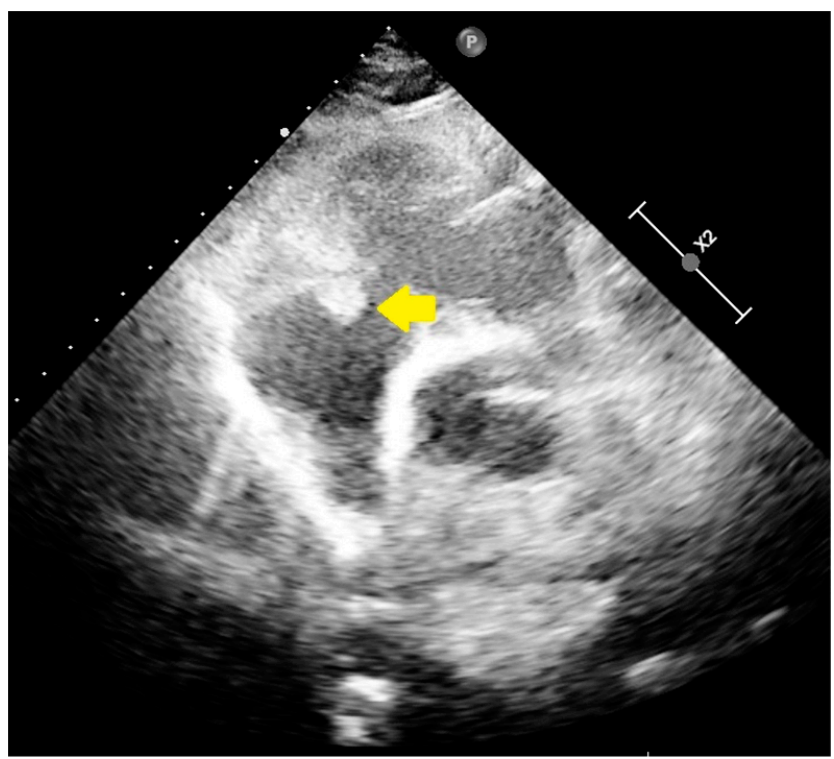

Figure 1. Transthoracic echocardiogram (TTE) showing infiltration by lymphoma in right atrium.

\section{Discussion}

Cardiac involvement by lymphoma may be primary or secondary. Primary cardiac lymphoma (PCL) is extremely rare, consisting in 1.3\% of all primary cardiac neoplasia [1]. In contrast, secondary involvement by lymphoma is a condition described in $20 \%$ of cases in studies post-mortem of patients with non-Hodgkin lymphoma but misdiagnosed at baseline [2]. According to the histological findings, primary and secondary cardiac lymphoma have been reported as NHL [3]. The symptom profile at baseline is often heterogeneous, including arrhythmias, pericardial tamponade or cardiogenic shock, which represent the most common causes of death. Several cases reported that clinical conditions at diagnosis are correlated with outcomes; in fact, patients with symptomatic heart failure show a worse outcome compared to those with arrhythmias or cardiac conduction abnormalities [1]. Although our patient had a high risk of early death, presenting a ventricular arrhythmia and hemodynamic instability at baseline, he was successfully treated thanks to the prompt diagnosis and rapid, but fractionated, therapy. The TTE examination was useful in identifying an atypical tissue infiltrating the myocardial muscle; the further CT scan showed the mediastinal mass and guided the subsequent mediastinum biopsy execution. The TTE is often the first-line screening imaging, characterized by a rapid and bedside approach and demonstrating $60 \%$ of sensitivity for the detection of cardiac involvement [4]. Cardiac NMR and cardio CT may also provide a specific anatomic assessment of cardiac involvement and are mandatory (both or single assessment) in this setting [5]. Advantages of CT include higher spatial resolution, three-dimensional volumetric coverage and a better ability to visualize epicardial coronary arteries and mediastinal structures, in particular lymph nodes, which are generally involved in patients with cardiac lymphoma [6]. Therefore, cardiac imaging can play a crucial role in these patients and may guide therapeutic management. In fact, we performed several TTEs during the treatment in order to monitor the safe reduction of the tissue infiltrating the myocardial muscle. Furthermore, not only the FDG$\mathrm{PET} / \mathrm{CT}$ but also the cardiac CT were performed at the end of treatment, thereby enabling a proper assessment of disease response to therapy [7]. Available data on survival of patients with cardiac involvement by lymphoma show an unfavorable outcome [1]. In the chemoimmunotherapy era, PCL seems to have a better prognosis compared to secondary cardiac lymphoma [1]. The literature data report a median survival of three months for patients with cardiac non-Hodgkin lymphoma. However, those who survive the first month of treatment achieve long-term disease control [1]. These data suggest the importance of an early chemotherapy treatment for these patients. In fact, we promptly started a treatment based on an R-COMP regimen, including a fractionated dose-dense scheme with $50 \%$ of the 
vincristine dosage, rituximab fractionated over three consecutive days, the half remaining dose of vincristine, followed by a full dose of cyclophosphamide and $50 \%$ of liposomal doxorubicin. A general consensus on how best to fractionate the initial chemotherapy doses in this subset of patients has not been achieved [8,9]. Nevertheless, according to baseline clinical characteristics, a patient-personalized approach is recommended to reduce potentially life-threatening risks, such as severe heart failure, ventricular fibrillation and cardiac rupture, in the early post-chemotherapy [10]. In particular, by aiming to obtain a safe tumor mass reduction of the full-thickness infiltration of myocardial tissue by lymphoma, we adopted a pre-phase therapy by VCR (fractionated over two days) followed by the rituximab administration (fractionated over three days). In addition, we decided to administer liposomal doxorubicin in consideration of its comparable efficacy and reduced cardiotoxicity in frail and elderly patients, which was demonstrated when replacing it for conventional doxorubicin in standard chemotherapy regimens for NHL [11].

\section{Conclusions}

In conclusion, we report a rare case of cardiac involvement by DLBCL, which benefited from a rapid multidisciplinary assessment and achieved CR in absence of complications. In addition, an innovative and personalized therapeutic approach was administered. The patient is still in CR at this time of writing after 24 months, in contrast with other published data [1]. Thus, we recommend the importance of a prompt and individualized chemotherapy based on fractionated therapy. Finally, we prove that strict monitoring of potential cardiac complications correlated with the tumor mass shrinking, improving the outcomes of these patients.

Author Contributions: S.M. wrote the manuscript. S.M., S.P., M.P.B., M.P., M.B., M.T. and A.T. were involved in the diagnosis and management of the patient. A.D.N. formulated histologic diagnosis. A.T. proposed and revised the case report. All authors have read and agreed to the published version of the manuscript.

Funding: This study received no specific grant from any funding agency in the public, commercial or not-for-profit sectors.

Institutional Review Board Statement: Not applicable.

Informed Consent Statement: Written informed consent was obtained from the patient to publish this paper.

Data Availability Statement: The data presented in this study are available on request from the corresponding author.

Conflicts of Interest: The authors declare no conflict of interest.

\section{References}

1. Gordon, M.J.; Danilova, O.; Spurgeon, S.; Danilov, A.V. Cardiac non-Hodgkin's lymphoma: Clinical characteristics and trends in survival. Eur. J. Haematol. 2016, 97, 445-452. [CrossRef] [PubMed]

2. McDonnell, P.J.; Mann, R.B. Involvement of the hearth by malignant lymphoma: A clinicopathologic study. Cancer 1982, 49, 944-951. [CrossRef]

3. Voigt, P.; Wienbeck, S.; Weber, M.A.; Oyama-Manabe, N.; Beimler, M.; Schob, S.; Kahn, T.; Meyer, H.J.; Randaxhe, J.F.; Surov, A. Cardiac Hematological Malignancies: Typical Growth Patterns, Imaging Features, and Clinical Outcome. Angiology 2017, 69, 170-176. [CrossRef] [PubMed]

4. Meng, Q.; Lai, H.; Lima, J.; Tong, W.; Qian, Y.; Lai, S. Echocardiographic and pathological characteristics of cardiac metastasis in patients with lymphoma. Oncol. Rep. 2002, 9, 85-88. [CrossRef] [PubMed]

5. Al-Mehisen, R.; Al-Mohaissen, M. Cardiac involvement in disseminated diffuse large B-cell lymphoma, successful management with chemotherapy dose reduction guided by cardiac imaging: A case report and review of literature. World J. Clin. Cases 2019, 2, 191-202. [CrossRef] [PubMed]

6. Maleszewski, J.J.; Bois, C.M. Neoplasia and the heart pathological review of effects with clinical and radiological correlation. JACC 2018, 2, 202-227. [CrossRef] [PubMed]

7. Meignan, M.; Itti, E.; Gallamini, A.; Younes, A. FDG PET/CT imaging as a biomarker in lymphoma. Eur. J. Nucl. Med. Mol. Imaging 2015, 42, 623-633. [CrossRef] [PubMed] 
8. Dawson, M.A.; Mariani, J.; Taylor, A.; Koulouris, G.; Avery, S. The successful treatment of primary cardiac lymphoma with a dose-dense schedule of rituximab plus CHOP. Ann. Oncol. 2006, 17, 176-177. [CrossRef] [PubMed]

9. Shah, K.; Shemisa, K. A "low and slow" approach to successful medical treatment of primary cardiac lymphoma. Cardiovasc. Diagn. Ther. 2014, 4, 270-283. [PubMed]

10. Ban-Hoefen, M.; Bernstein, S.H. Symptomatic intracardiac diffuse large B-cell lymphoma. Am. J. Hematol. 2009, 84, 683-685. [CrossRef] [PubMed]

11. Zinzani, P.L.; Federico, M.; Oliva, S.; Pinto, A.; Rigacci, L.; Specchia, G.; Tucci, A.; Vitolo, U. The more patients you treat, the more you cure: Managing cardiotoxicity in the treatment of aggressive non-Hodgkin lymphoma. Leuk. Lymphoma 2014, 56, 12-25. [CrossRef] [PubMed] 